\title{
AMPLIANDO O CAMPO DA ATENÇÃO PSICOSSOCIAL: A ARTICULAÇÃO DOS CENTROS DE ATENÇÃO PSICOSSOCIAL COM A SAÚDE DA FAMÍLIA
}

\author{
Extending the Field of the Psychosocial Attention: \\ The J oint of the Centers of Psychosocial Attention with the Health of the Family \\ Ampliando el Campo de la Atención de Psicosocial: \\ La Articulación de los Centros de Atención Psicosocial con la Salud de la Familia
}

Ândréa Cardoso de Souza

\begin{abstract}
Resumo
Trata-se de uma reflexão sobre a articulação entre o Centro de Atenção Psicossocial e as equipes de Saúde da Familia no que se refere à ampliação das ações no campo da saúde mental. Tem como objetivo discutir a implementação da política de saúde mental no âmbito da atenção básica de saúde. Para tanto, foi realizada uma pesquisa bibliográfica. Entre os resultados, observa-se que a orientação das políticas de atenção à saúde mental aponta para a consolidação de novas estratégias de cuidado à medida que propõe redirecionar as ações para serviç̧os de base territorial. Conclui-se que tanto a Saúde da Família quanto os Centros de Atenção Psicossocial constituem dispositivos privilegiados para transformaç̃̃es das práticas de atenção em saúde mental. Possibilitam a construção de outras formas de convivência com as diferenças, ampliando as redes de solidariedade em um dado território.
\end{abstract}

Palavras-chave: Saúde Mental. Saúde da Família. Serviços de Saúde Mental.

\begin{abstract}
It is a reflection about the joint between the Center of Psychosocial Attention and the staff of Family Health in what is related to the magnifying of the actions in the mental health field. It has as objective to argue the implementation of the mental health politics in the scope of the basic attention of health. For in such a way, a bibliographical research was carried through. Among the results, it is observed that the orientation of the politics of attention to the mental health points to the consolidation of new strategies of care as consider redirect the actions for services of territorial basis. It was concluded that as much for the Family Health as for the Centers of Psychosocial Attention constitute privileged devices for transformations of the practices of attention in mental health. They make possible the construction of other forms of coexistence with the differences, extending the net of solidarity in a determined territory.
\end{abstract}

\begin{abstract}
Resumen
Tratase de una reflexión sobre la articulación entre el Centro de Atención Psicosocial y el equipo de Salud de la Familia en qué se refiere en la ampliación de las acciones en el campo de la Salud Mental. Tiene como objetivo discutir la implementación de la política de salud mental en el ámbito de la atención básica de salud. Para tanto, fue realizada una investigación bibliográfica. Entre los resultados, se observa que la orientación de las políticas de atención a la salud mental señala la consolidación de nuevas estrategias de cuidado conforme propone redireccionar las acciones para los servicios de base territorial. Concluyese que tanto la Salud de la Familia como para los Centros de Atención Psicosocial constituyen dispositivos privilegiados para transformaciones de las prácticas de atención en salud mental. Hacen posible la construcción de otras formas de convivencia con las diferencias, extendiendo la red de solidaridad en un territorio determinado.
\end{abstract}

Keywords:

Mental Health. Family Health. Mental Health Services.
Palabras clave:

Salud Mental. Salud de la Familia. Sevicios de Salud Mental. 
EM TEMPOS DE TRANSIÇÃO:

O VELHO NÃO MAIS DOMINA E O NOVO AINDA NÃO PREDOMINA

Torna-se premente a desconstrução do modelo assistencial tradicional no campo da saúde, definido pelo Ministério da Saúde como atendimento da demanda espontânea, eminentemente curativo, hospitalocêntrico, de alto custo, sem instituir redes hierarquizadas por complexidade, com baixa resolutividade. Nele, a equipe de saúde não estabelece vínculos de cooperação e de co-responsabilidade com a comunidade.

Se não forem produzidas mudanças nos pressupostos e paradigmas que norteiam o modelo assistencial tradicional, não se poderá esperar resposta satisfatória dos modos de atenção. Para isso, será necessário desconstruir e reconstruir o modelo, e essa reconstrução deve considerar a clínica do sujeito ${ }^{1}$, que inclui a doença, o contexto e o próprio sujeito. Para que a transformação desse modelo ocorra, são necessárias mudanças na concepção e na forma de se trabalhar em saúde. É preciso reformular a clínica, tomando não apenas a doença, mas o sujeito enfermo, ampliar as práticas de promoção da saúde, atuar em equipe interdisciplinar, ampliar espaços para que se atenda além do consultório e da sala de procedimentos, isto é, no domicílio, na escola, no bairro, na rua, etc, e sobretudo cuidar das pessoas, inserindo-as em redes sociais, qual seja, o território, a família, etc".

Os serviços de saúde devem abandonar a lógica tradicional que rege seu funcionamento e buscar criar condições para que o sistema de saúde se aproxime mais dos indivíduos, tornando-se mais humano, solidário e, sobretudo, mais resolutivo.

Durante a implementação do movimento de Reforma Sanitária, observou-se a crescente consciência social de que a luta pela saúde faz parte da construção da cidadania. Um dos produtos desse movimento foi a implantação do Sistema Único de Saúde (SUS). Entretanto, há que se considerar outros ganhos, visto que esse movimento se configurou como um projeto social em busca da reorientação do modelo assistencial, através de estratégias como a da Saúde da Família (SF) e os Centros de Atenção Psicossocial.

Tanto o movimento da Reforma Sanitária quanto o da Reforma Psiquiátrica partem de princípios e diretrizes que vislumbram a construção de formas mais humanas e mais acolhedoras de se pensar e de se promover saúde, implicando a elaboração de novos modos de atenção que contemple essa perspectiva mais ampliada. Este artigo procura levantar pontos considerados essenciais para a realização de discussão que tenha como foco a implementação de políticas de saúde mental no âmbito da atenção básica, em especial no que concerne à inclusão de ações em saúde mental na SF.

0 referencial teórico utilizado encontra-se na interface entre Reforma Sanitária e Reforma Psiquiátrica. A proposta metodológica privilegiou a análise qualitativa. Este artigo consiste numa pesquisa bibliográfica de artigos científicos dos últimos cinco anos, nas bases de dados MEDLINE e LILACS, disponíveis na Bireme e através da SCIELO e busca eletrônica na biblioteca da ENSP e da UNICAMP, no período de agosto de 2003 a julho de 2004.0s artigos foram selecionados tendo como referência a temática saúde mental e saúde da família.

\section{A REFORMA PSIQUIÁTRICA EM MOVIMENTO}

0 movimento da Reforma Psiquiátrica propunhase a resgatar a história, a tornar possível a vida das pessoas que viviam confinadas no interior dos hospitais psiquiátricos, além de impelir o sistema de saúde a elaborar modos mais humanizados e dignos de cuidado. Como movimento social, ampliou a pauta de discussões e reivindicações e propôs a superação radical do modelo psiquiátrico tradicional, utilizado nas práticas assistenciais e que representava a soberania do saber médico sobre a loucura. Buscava desenvolver estratégias que possibilitassem a ampliação das fronteiras de atuação para além dos bem demarcados limites dos hospitais psiquiátricos e das universidades.

Este movimento propiciou um intenso debate sobre as novas exigências políticas e éticas no contexto das transformações assistenciais da nova política nacional de Saúde Mental. 0 principal objetivo da Reforma Psiquiátrica constitui-se em poder transformar as relações que a sociedade, os sujeitos e as instituições estabeleceram com a loucura, com o louco e com a doença mental, conduzindo tais relações no sentido da superação do estigma, da segregação e da desqualificação dos sujeitos, ou, ainda, estabelecer com a loucura uma relação de coexistência, de troca, de solidariedade, de positividade e de cuidados $^{2}$.

A Atenção Psicossocial, ao adotar o princípio de saúde como direito e como norteador das práticas, visa a garantir uma atenção digna, acolhedora, singular e responsável a todas as pessoas que se encontram em sofrimento psíquico. No centro da mudança no campo da saúde mental, está o desafio de cuidar sem segregar.

Hoje, a política nacional de saúde mental reforça a atenção de base territorial, em substituição à atenção hospitalar tradicional. Essa reorientação pode ser observada na diminuição do total de leitos psiquiátricos e no aumento do número de Centros de Atenção 
Psicossocial (CAPS) no país. Pode-se observar uma tendência à inversão do modelo de assistência em saúde mental, havendo uma retração do modelo hospitalar e um avanço do modelo de atenção psicossocial. ${ }^{3}$

No contexto da crise do paradigma psiquiátrico, vão sendo construídas novas experiências e novas propostas de intervenção no campo da saúde mental. A psiquiatria clássica entra em um processo de falência de seus dispositivos teórico-práticos a partir do momento em que se instaura uma transformação radical no objeto e, também, uma ruptura epistemológica do saber psiquiátrico: o foco da intervenção da psiquiatria deixa de ser o tratamento da doença mental para constituirse na promoção da saúde mental. ${ }^{4}$

Consideramos a Reforma Psiquiátrica um processo social complexo e que possui quatro dimensões que devem ser trabalhadas de forma simultânea e interrelacionada ${ }^{5}$, quais sejam:

- Dimensão teórico-conceitual - refere-se ao campo epistemológico, aos conceitos e saberes que respaldam as práticas desenvolvidas no âmbito da saúde mental.

- Dimensão técnico-assistencial - relaciona-se com os modos de atenção em saúde mental e com os conceitos e as práticas advindas deste paradigma. - Dimensão jurídico-política - propõe-se a discutir questões relacionadas à revisão da legislação sanitária e psiquiátrica, resgatando para a pauta das discussões questões como cidadania, direitos (civis, humanos e sociais), ao mesmo tempo em que aponta para a necessidade de redefinição das relações sociais até então estabelecidas com a loucura e com o sujeito em sofrimento psíquico.

- Dimensão sociocultural - propõe-se a trabalhar na perspectiva de transformação do imaginário social acerca da loucura, vislumbrando um outro lugar para a loucura e para o sujeito em sofrimento psíquico na sociedade. Esta dimensão é a que apresenta maior dificuldade para ser alcançada, em face de toda história estigmatizante da loucura e pela relação de dependência com as demais dimensões, de forma integrada e articulada, assegurando no cotidiano da vida outros significantes para a loucura. É ela que contempla o objetivo maior da Reforma Psiquiátrica, que é o de promover transformações sociais acerca do imaginário social da loucura.

A Reforma Psiquiátrica implica mudanças culturais e sociais radicais nesses quatro campos, afirmandose como movimento social, o que nos permite avançar e descobrir novos conceitos, outras práticas, outros lugares tanto para os usuários quanto para os técnicos de saúde mental. A articulação entre os Centros de Atenção Psicossocial (CAPS) e a Saúde da Família (SF) pode viabilizar essas transformações tão caras ao processo da Reforma Psiquiátrica.

A Reforma Psiquiátrica propõe o abandono do lugar zero de trocas sociais (o manicômio) e a assunção de um lugar múltiplo, diverso, que é o território ${ }^{6}$. Para isto, faz-se necessário reafirmar a necessidade de intervir na sociedade, pois é na sociedade que se originam mecanismos de estigmatização e de exclusão das diferenças ${ }^{7}$. A Reforma Psiquiátrica também propõe a criação de outros espaços possíveis para a loucura e o direcionamento da intervenção para espaços sociais.

\section{CAPS: PRÁTICAS PARA ALÉM DOS SERVIÇOS}

0 processo de Reforma Psiquiátrica apontava para a construção de novos modos de atenção em saúde mental que proporcionassem a ruptura com 0 aparato institucional criado para "conter" a loucura. Assim, em alternativa ao modelo hospitalar de atenção, a priori excludente, foram implantadas diversas experiências que visavam a transformar as práticas e saberes relacionados a este campo, em que se pretendia, entre outros, a construção de novos modos de atenção em saúde mental que se diferenciasse do que hegemonicamente era oferecido. Entre essas experiências, podemos citar a reorientação da política nacional de saúde mental do eixo hospitalar para o eixo territorial, o que inclui a construção de serviços de atenção diária, serviços residenciais terapêuticos, centros de convivência, entre outros. Podemos citar também a inclusão das ações de saúde mental na atenção básica, principalmente na região nordeste do país ${ }^{8}$.

Os CAPS surgem, no Brasil, em fins da década de 80, como um análogo, ainda incipiente, dos Centros de Saúde Mental italianos, no que tange a oferecer um cuidado intensivo ao portador de sofrimento psíquico a partir de uma lógica de inserção no território, vínculo, acolhimento e atenção diária. Os CAPS ocupam atualmente no Brasil um papel estratégico para a transformação das práticas de atenção em saúde mental $\left.\right|^{9}$.

Esses novos serviços podem ser denominados espaços sociais de produção de sujeitos sociais e de subjetividades, espaços de convivência, de sociabilidade de solidariedade e de inclusão. Os serviços substitutivos em saúde mental representam uma inovação em um contexto de prática médica tradicional.

0 termo CAPS foi pela primeira vez utilizado pela Secretaria Estadual de Saúde de São Paulo para 
descrever um serviço de atenção diária com funcionamento em dois turnos, durante cinco dias na semana, que pretendia ser intermediário entre a unidade hospitalar e a comunidade. Foi definido na época como um espaço de referência para o usuário - lugar para ir, encontrar pessoas, desenvolver alguma atividade, conversar, tomar um lanche, tomar remédio e ir para casa ${ }^{10}$. Entre seus objetivos estava o de constituir-se como "continente" para as pessoas em sofrimento psíquico, auxiliando-as no seu cotidiano. Constituía uma estratégia de cuidado para uma clientela marginal e um suporte adequado à complexidade dos aspectos que envolviam o sofrimento psíquico, não encontrado nos dispositivos existentes até então ${ }^{9}$.

0 Ministério da Saúde adotou o termo CAPS para descrever os serviços tidos como substitutivos que se tornaram estratégia de enfrentamento ao modelo assistencial tradicional. Atualmente, os CAPS são definidos pelo Ministério da Saúde como um serviço ambulatorial de atenção diária que funciona segundo a lógica do território. Representam o articulador central das ações de saúde mental do município ou do módulo assistencial e se apresentam nas seguintes modalidades de serviços: CAPS I, CAPS II e CAPS III, CAPS infanto-juvenil e CAPS álcool e drogas, definidos por ordem crescente de porte e complexidade e abrangência populacional ${ }^{11}$.

Nos dez anos transcorridos entre a publicação da primeira regulamentação e sua republicação em 2002, a cartografia da Atenção Psicossocial, no Brasil, apresentou importantes transformações no que diz respeito à oferta de Centros de Atenção Psicossocial e seu papel na rede de saúde mental.

0 CAPS pode ser definido como uma experiência em saúde coletiva, tendo como objetivo interferir na produção do imaginário social no que diz respeito à loucura. Juntamente com outros dispositivos de atenção territorial, a sociedade, que durante toda a existência da psiquiatria pensava que o melhor tratamento e encaminhamento destinado ao louco seria o hospital psiquiátrico, agora está conhecendo outros modos de lidar com a loucura que não a segregação e a exclusão do portador de doença mental.

Neste sentido, o CAPS apresenta-se como substitutivo do hospital psiquiátrico e centra-se na atenção integral ao portador de sofrimento psíquico. Portanto, visa ao desenvolvimento de projetos de vida, de produção social e à promoção da melhora da qualidade de vida dos usuários. Tem como missão estabelecer laços para além dos serviços de saúde, por isso deve estar voltado para "fora de si", pois precisa manter aquecida sua relação com a cultura local.
Apesar de buscar, através do desenvolvimento de suas práticas, uma permeabilidade com o social, o CAPS apresenta dificuldades em coaabitar o espaço de fora, por diversos e diferentes motivos (estrangulamento da rede, aumento da demanda no CAPS para acompanhamento ambulatorial, formação ainda "inadequada" para esta nova proposta de atenção, dentre outras), sendo cada vez mais distante a possibilidade de o CAPS estar fora de seus muros.

É necessário ampliar a projeção do CAPS diante das políticas sociais. Para tanto, é essencial poder estruturá-lo de forma a ocupar outros territórios - algo muito caro e desejável no campo da atenção em saúde mental. 0 CAPS precisa se inscrever de maneira mais ampla na transformação social.

0 CAPS, ao buscar inovação em suas práticas, não pode prescindir do resgate de sua origem: o conceito de território como processo, a tomada de responsabilidade sobre a demanda, o conceito de desinstitucionalização, a dimensão cultural e ética do cuidado em saúde mental e, principalmente, a compreensão da transformação do modelo assistencial em saúde mental como um processo social.

Diferente do hospital psiquiátrico que impunha uma barreira ao intercâmbio social e às possibilidades de troca com o mundo exterior, o CAPS busca, deseja e de certa forma corre ao encontro da "contaminação" ou da "invasão" da loucura na sociedade. Através da SF, o CAPS tomado aqui como conceito operacional ${ }^{12}$, pode ocupar as ruas, a vizinhança, o território, capilarizando-se e sendo capaz de operar mudanças nas microrelações que se dão no cotidiano.

A SF poderia constituir um mediador de encontros, de saberes e práticas "não-especializadas", de espaços não delimitados para a circulação da loucura. $\mathrm{Na}$ tentativa de se trabalhar com outras perspectivas, de se criar novos modos de acolher a saúde e a loucura, as ações deixam de ser restritas a espaços físicos, possibilitando um trânsito até então considerado "inseguro". Torna-se difícil deixar de lado a "segurança" do consultório ou da unidade de saúde e, de certa forma, habitar o espaço até então do outro ${ }^{8}$.

0 estabelecimento de ações em conjunto entre a Saúde Mental e a Estratégia de Saúde da Família amplifica o potencial do CAPS como agenciador de novos modos de cuidado e estende a outros espaços a responsabilização pelo cuidado integral às pessoas em sofrimento psíquico. 0 CAPS pode funcionar como unidade de apoio para as equipes de Saúde da Família ou da Atenção Básica, porém são estas equipes que continuarão sendo referência para as pessoas. Não haveria transferência de responsabilidade, o CAPS 
funcionaria como uma unidade de apoio que viabilizaria melhor atenção às pessoas, prestada pelas equipes de Saúde da Família , caminhando no sentido da integralidade da atenção. Fazer saúde mental na rua, na comunidade, no bairro implica deixar de lado a regularidade mais ou menos segura dos serviços e ficar diante da imprevisibilidade radical da vida cotidiana. Portanto, não é tarefa fácil colocar-se diante do novo, da vida das pessoas, no meio dos acontecimentos; entretanto, é lá que é preciso estar, pois é lá que as coisas acontecem ${ }^{13}$.

\section{A SAÚDE DA FAMÍLIA: UMA ESTRATÉGIA EM BUSCA DA INTEGRALIDADE}

A SF tem sido a principal estratégia adotada pelo Ministério da Saúde para a reorganização da Atenção Básica, buscando o acompanhamento permanente da saúde de um número determinado de indivíduos e famílias que residem em um território. Possibilita o estabelecimento de vínculos de compromisso e de coresponsabilidade entre profissionais de saúde e a população, contribuindo para a estruturação dos demais níveis de complexidade do sistema de saúde, de forma que se mantenha o compromisso com o acesso da população a todos os níveis de assistência e com a implantação de novos modos de atenção ${ }^{14}$.

A SF pode ser considerada um espaço de práticas de um novo modo de conceber o sistema de saúde, uma estratégia para o alcance da eqüidade e da integralidade em saúde. Representa a transformação de um discurso ideológico em uma ação política, que tem como objetivo a reorganização da prática assistencial em novas bases e critérios ${ }^{15}$, em substituição ao modelo tradicional de assistência, constituindo novos vínculos entre os serviços de saúde e a população. Promover a saúde de um indivíduo implica intervir no contexto onde o mesmo está "inserido", no espaço onde ele vive. A atenção passa a ser centrada na família, percebida a partir de seu ambiente físico e social, o que tem permitido às Equipes de Saúde da Família uma compreensão ampliada do processo saúde/doença e da necessidade de intervenções que vão além das práticas curativas ${ }^{16}$.

A SF vem se colocando cada vez mais como estruturante para a reorganização dos serviços de atenção básica e para a reorientação das práticas em saúde, na tentativa de inverter a concepção atual do modelo tradicional vigente, considerando a saúde como produto social. No entanto, torna-se necessário que a equipe de saúde desempenhe o papel de agente transformador ${ }^{17}$, estabelecendo vínculos afetivos e efetivos de cooperação com os indivíduos, famílias e comunidade.
Poderíamos então afirmar que a SF se configura em uma política democratizadora, em contraposição ao caráter racionalizador das políticas anteriores. Caracteriza-se como uma prática de atenção de base epidemiológica, ancorada em dois conceitos da medicina social - o da determinação social do processo saúde-doença e o do enfoque nos processos de trabalho em saúde. Tem como características o caráter de organização de serviços, a potencialidade de substituição do modelo assistencial tradicional e o fato de constituir fonte de inspiração para novas práticas de gestão ${ }^{16,18}$. Esta impele para a necessidade da participação e para novas e diferentes maneiras de organizar a atenção à saúde na localidade.

Nesse sentido, consideramos a SF uma estratégia altamente potente na promoção de outros modos de relacionamento com a loucura, desconstruindo e construindo no interior das casas, na vizinhança, na comunidade, no bairro outras relações com as diferenças, permitindo ao portador de doença mental um espaço de circulação que não aquele "especializado", segregador. Por meio de um atendimento "não especializado", podemos pensar e traçar novos trajetos e circuitos para a loucura e trabalhar para desmistificar a loucura no cotidiano, permitindo ao sujeito em sofrimento psíquico compartilhar o mesmo espaço que as demais pessoas na unidade de saúde, na associação de bairro, no trabalho, lazer, etc. Dessa forma, é possível haver outro trânsito, que poderá apontar para a tão almejada transformação social e permitir à loucura e ao sujeito em sofrimento coabitar num espaço múltiplo, atravessado por singularidades, diferenças e conflitos, portanto, permitir ao portador de doença mental um lugar, de fato, na vida.

\section{CONSIDERAÇÕES FINAIS}

A SF, por meio do desenvolvimento de suas ações, pode proporcionar o estabelecimento de relações consistentes com a comunidade, operando em seu interior as tão almejadas mudanças e transformações sociais, e, talvez por isso, pode ser o dispositivo que melhor venha atender aos objetivos da Reforma Psiquiátrica, pois pode viabilizar a construção de outras representações e lugares para a loucura. É preciso estar dentro da comunidade e não ao lado, pois o processo de transformação acontece na comunidade. Para tanto, necessitamos estabelecer processos contínuos de apoio mútuo, articulando recursos, pessoas e instituições, para podermos vislumbrar a possibilidade de mudanças nos mais diferentes campos. Desse modo, a SF poderia operar melhor o princípio da acessibilidade, tão caro à Atenção Psicossocial ${ }^{19}$, por meio dos agentes comunitários, atuando próximo 
das pessoas, na vizinhança e fazendo a extensão de suas práticas na casa das pessoas.

Essa articulação possibilita tanto à SF quanto aos CAPS tornar as fronteiras permeáveis, levando-nos a percorrer caminhos diferentes, propiciando ao portador de transtorno mental sair de um lugar segregador e excludente para de agenciamentos de possibilidades de vida. Permite tornar concretos, no cotidiano da vida das pessoas, os princípios que motivaram e nortearam o movimento tanto da Reforma Psiquiátrica quanto da Reforma Sanitária. Essa articulação possibilita e requer maior plasticidade das práticas de atenção em saúde.

Talvez, a SF nos permita de fato transformar, mudar, reformar, reformular, revolucionar os saberes e práticas no campo da saúde mental ${ }^{20}$ e, por conseguinte, avançar na elaboração de novos conceitos e práticas pertinentes ao campo da Atenção Psicossocial, fundamentados pelos princípios da Reforma Psiquiátrica.

Essa articulação pode proporcionar a formulação de novas tecnologias de atenção, de teorias e práticas, e aí se caracterizar como um dispositivo inovador, pois a idéia de inovação traz em si um caráter de ruptura com 0 já estabelecido ${ }^{21}$. Pode possibilitar a aproximação com a clínica criadora de possibilidades, produtora de sociabilidades e subjetividades ${ }^{3}$, ou ainda trabalhar na perspectiva de uma clínica antimanicomial ${ }^{22}$, que convide o sujeito a sustentar sua diferença, sem precisar excluirse do social, que introduz a idéia de que "fazer caber" o louco na cultura é também ao mesmo tempo convidar a cultura a conviver com certa falta de cabimento, reinventando com ela também seus limites. Pode fazer pensar, propor, sustentar formas de contrato social nas quais a diferença possa ser incluída.

Talvez tal "desorganização" propiciada pela SF, como estratégia de ação, poderia possibilitar à comunidade novas formas de convivência com a loucura, nas quais as diferenças pudessem ser atravessadas.

Tal articulação enseja a ruptura com "antigos" padrões assistenciais e aponta para a superação da racionalidade médica, além de buscar a produção de novos modos de atenção coerentes com os princípios do SUS (universalidade, eqüidade, integralidade, resolutividade, intersetorialidade, humanização do atendimento e participação social), podendo implementar práticas que de fato atendam aos princípios de ambas as políticas públicas de saúde. Trabalhando em conjunto, podem proporcionar uma atenção integral, compartilhar a responsabilidade pela melhoria da qualidade de saúde e de vida de um dado território e tornar os dispositivos de atenção mais acessíveis àqueles que deles necessitam. Em rede, é possível potencializar a capacidade de produzir mudanças. Articulações como essa podem apontar para a possibilidade de construirmos outros modos de promover a saúde e de colocar em prática, ainda que tardiamente, os preceitos do Sistema Único de Saúde brasileiro.

A SF poderia operar como dispositivo privilegiado da saúde mental para promover a inserção das pessoas em um território de abrangência, contribuindo para a transformação cultural da sociedade em relação à loucura, dado o modo de desenvolvimento de suas ações e tornando-se um dispositivo importante na Atenção Psicossocial, devido ao fato de que muitas pessoas se encontram em situação de institucionalização dentro de suas próprias casas. Sendo assim, a SF teria maior possibilidade de acesso e de vinculação a essas pessoas.

Essa articulação facilitaria ao CAPS circular pelos espaços de vida das pessoas, algo fundamental para a implementação destas novas práticas. 0 CAPS deve atuar no território, nos espaços e percursos que compõem a vida das pessoas ${ }^{6}$, pois é na sociedade que funciona os mecanismos originários de marginalização do doente mental. Essa articulação, de fato, pode contribuir para a construção social de outros modos de lidar com a loucura.

Para que a SF se constitua como uma política de reorientação da atenção à saúde é fundamental que se tenha uma equipe comprometida com os princípios do SUS, para que possa operar como diferenciador, não reproduzindo as formas de "funcionar" do modelo tradicional. Seria necessário também, que o mesmo agregasse características como diversidade, ousadia, criatividade e resolutividade, para que seja possível trabalhar na perspectiva da (re)organização da atenção à saúde. Neste sentido, a SF tem incorporado alguns conceitos trabalhados pela saúde mental, como responsabilização pela demanda, estabelecimento de vínculos e acolhimento. Outra concepção que envolve tanto a SF quanto a política de saúde mental é a de que as ações precisam se dar onde as pessoas estão, experimentando seus estilos de vida, na trama de possibilidades, funções, papéis, trabalhos, lazeres e sofrimento, contribuindo assim para a construção de uma outra prática de atenção à saúde. Tanto uma quanto outra prevêem uma estreita relação do setor saúde com outros setores da sociedade, pois, para que se constituam práticas diferenciadas, devem se integrar como uma rede de relações que extrapolem o âmbito da saúde (em um conceito restrito), e devese trabalhar para a construção de relações sociais em busca de uma nova atitude diante da complexidade do processo de saúde. Ambas primam pela elaboração de outras formas de organização do cotidiano que não 
passe exclusivamente pela agenda de atendimentos, permitindo acolher as "emergências" do dia-a-dia.

A saúde mental e a saúde da família têm ampliado suas possibilidades de atuação, transformando as práticas vigentes, tendo como lócus de ação o território. Como políticas públicas, visam ao deslocamento dos cuidados de crise, de emergência, do manejo de situações graves, para o espaço da residência, envolvendo família e vizinhança.

A articulação da saúde mental com a SF poderia gerar maior "tolerância" às diferenças, maior confiança e ampliar as redes de solidariedade entre moradores de um determinado local, contribuindo para o desenvolvimento de mudanças culturais. Talvez a SF possa viabilizar a construção de laços sociais entre a comunidade e a loucura mais consistentes que o CAPS. Poderíamos dizer que 0 CAPS e a SF se interdependem e se interpotenciam ${ }^{23}$. A SF seria, então, a principal estratégia para viabilizar a integração das aç̃̃es de saúde mental na atenção básica.

Para a transformação do modelo assistencial, é preciso que os técnicos considerem os valores e a cultura da comunidade; além disso, torna-se necessário o estabelecimento de parcerias, sem as

\section{Referências}

1. Campos GWS. Saúde Paidéia. São Paulo (SP): Hucitec; 2003.

2. Amarante P. Loucura, cultura e subjetividade: conceitos e estratégias, percursos e atores da reforma psiquiátrica brasileira. In: Fleury $S$, organizadora. Saúde e democracia: a luta do CEBES. São Paulo (SP): Lemos Ed; 1997.

3. Freire FHMA.. O Sistema de Alocação de Recursos do SUS aos Centros de Atenção Psicossocial: implicações com a proposta de atuação dos serviços substitutivos [dissertação de mestrado]. Rio de Janeiro (RJ): Escola Nacional de Saúde Publica/FIOCRUZ; 2004.

4. Organização Pan-americana de Saúde - OPAS. Declaração de Caracas. Caracas (Venezuela), 1990.

5. Amarante P. Clínica e a reforma psiquiátrica. In: Amarante P, organizador. Archivos de saúde mental e atenção psicossocial. Rio de janeiro (RJ): NAU; 2003.

6. Kinoshita RT. Em busca da cidadania. In: Campos FCB, Henriques CMP, organizadoras. Contra a maré a beira mar. São Paulo (SP): Hucitec; 1997.

7. Desviat M. A reforma psiquiátrica. Rio de Janeiro(R)): Ed da FIOCRUZ; 1999.

8. Jatene A, Lancetti A. Saúde Loucura, 7. Saúde mental e saúde da família. $2^{a}$ ed. São Paulo (SP): Hucitec; 2001.

9. Guljor APF. Os Centros de Atenção Psicossocial: um estudo sobre a transformação do modelo assistencial em saúde mental [dissertação de mestrado]. Rio de Janeiro (RJ): Escola Nacional de Saúde Publica/ FIOCRUZ; 2003.

10. Yasui S. CAPS: aprendendo a perguntar. In: Lancetti A, organizador. Saúde Loucura, 1. São Paulo (SP): Hucitec; 1989. quais não há mudança possível. É necessário buscar integrar esses dois campos para oferecermos de fato uma atenção mais integral às pessoas.

Consideramos a SF mais um dispositivo de atenção para alcançar o objetivo fim da Reforma Psiquiátrica, que é o de promover mudanças culturais e sociais em relação à loucura. Poderíamos pensar na SF como um dispositivo altamente potente para promover tais mudanças.

Se não houver ampla mobilização e compromisso ético-político de planejadores, gestores e trabalhadores sobre essa inversão do modelo assistencial, corre-se o risco de que experiências promissoras, como a da saúde mental na atenção básica, reproduzam a lógica de um cuidado medicalizante, de exames e padrões de diagnósticos e de encaminhamentos pouco resolutivos.

Talvez, um dos maiores desafios dessa articulação seja "capacitar" tanto as equipes de SF quanto a dos novos serviços de saúde mental para um pensar e agir voltado para a Atenção Psicossocial, sem recorrer às "velhas" estruturas, e construir no dia-a-dia novos modos que possam efetivamente auxiliar as pessoas a cuidarem de si mesmas e andarem suas vidas.

11. Portaria $n^{0} 336$ de 19 de fevereiro de 2002. Atualiza a regulamentação dos serviços de atenção diária, estabelecendo modalidades de serviços: definidos por ordem crescente de porte/ complexidade e abrangência populacional. Brasília(DF): Ministério da Saúde.Secretaria de Assistência à Saúde; 2002.

12. Alves DS. Integralidade nas políticas de saúde mental. In: Pinheiro R, Mattos RA, organizadores. Os sentidos da integralidade na atenção e no cuidado à saúde. Rio de Janeiro (RJ): IMS/UERJ/ABRASCO; 2001.

13. Souza AC. Em tempos de PSF: novos rumos para atenção em saúde mental?. [dissertação de mestrado]. Rio de Janeiro (RJ): Escola Nacional de Saúde Pública /FIOCRUZ; 2004.

14. Portaria $n^{\circ} 648$, de 28 de março de 2006 . Estabelece a revisão de diretrizes e normas para a organização da Atenção Básica, para 0 Programa de Saúde da Família e para o Programa de Agentes Comunitários de Saúde- PACS e institui a Política Nacional de Atenção Básica. Brasília (DF): Ministério da Saúde. Secretaria de Assistência à Saúde; 2006.

15. Campos GWS. Subjetividade e administração de pessoal: considerações sobre modos de gerenciar o trabalho em equipes de saúde. In: Merhy E, Onoko R, organizadores. Agir em saúde: um desafio para o público. São Paulo (SP): Hucitec; 1997.

16. Merhy EE. A rede básica como uma construção da saúde pública e seus dilemas. In: Merhy E, Onoko R, organizadores. Agir em saúde: um desafio para o público. São Paulo (SP): Hucitec; 1997.

17. Gomes MCPA, Pinheiro R. Acolhimento e vínculo: práticas de integralidade na gestão do cuidado em saúde em grandes centros urbanos. Interface: comunicacão, saúde e educação 2005 mar/ago; 9(17): 287-301.

18. Goulart FAA. Experiência em Saúde da Família: cada caso é um caso? [tese de doutorado]. Rio de Janeiro (RJ): Escola Nacional de Saúde Pública/FIOCRUZ; 2002. 
19. Costa-Rosa A. 0 Modo psicossocial: um paradigma das práticas substitutivas ao modo asilar. In: Amarante P, organizador. Ensaiossubjetividade, saúde mental e sociedade. Rio de Janeiro (RJ): Ed da FIOCRUZ; 2000.

20. Rosa WG, Labate RC. A contribuição da saúde mental para o PSF. Rev Bras Enferm 2003 maio/jun; 56(3): 230-33.

21. Tanaka 0, Melo C. Inovação e gestão: a organização social no setor saúde. São Paulo (SP): Annablume / FAPESP; 2002.

22. Lobosque AM. Experiências da loucura. Rio de Janeiro (RJ): Garamond; 2001.

23. Sampaio JJC, Barroso CMC. Centros de Atenção Psicossocial e Equipes de Saúde da Família: diretrizes e experiências do Ceará. In: Jatene A, Lancetti A, organizadores. Saúde Loucura, 7. Saúde mental e saúde da família. $2^{a}$ ed. São Paulo(SP): Hucitec; 2001.

\section{Sobre a Autora}

\section{Ândréa Cardoso de Souza}

Enfermeira, Professora da Escola de Enfermagem Aurora de Afonso Costa/UFF, Mestre em Saúde Pública - ENSP/FIOCRUZ e Especialista em Saúde Mental e Psiquiatria - UNI-RIO 